\title{
Hemoparásitos Presentes en Poblaciones Ferales de la Paloma de Castilla (Columba livia) en el Departamento de Lima, Perú
}

\author{
Hemoparasites Present in Feral Populations of Rock Dove (Columba livia) in the \\ Department of Lima, Peru \\ Nancy Carlos Erazo ${ }^{1,3}$, Freddy Arellano Lima ${ }^{2}$, Nidia Puray Chavez ${ }^{2}$, \\ Adela Barraza Hernández ${ }^{2}$, Paloma Alcázar García ${ }^{1}$
}

\section{Resumen}

El objetivo del presente estudio fue determinar la presencia de hemoparásitos en poblaciones ferales de la paloma de castilla (Columba livia) en el departamento de Lima, Perú. Se trabajó en el centro poblado Pampas San Alejo, ciudad y provincia de Barranca (zona rural) y en un Zoológico del distrito de San Juan de Miraflores, ciudad y provincia de Lima (zona urbana). Se capturaron 52 aves adultas utilizando redes de neblina: 28 y 24 aves en la zona rural y urbana, respectivamente. Se obtuvo una muestra sanguínea de la vena braquial y se realizaron frotices sanguíneos, que fueron fijados con metanol y teñidos con Giemsa. El 94.2\% (49/52) de las aves fueron positivas a la presencia de hemoparásitos: Haemoproteus sp $(94.2 \%$; 47/52), Plasmodium sp $(13.5 \%$; 7/52) y Leucocytozoon sp $(1.9 \% ; 1 / 52)$. La intensidad de infección para Haemoproteus sp fue muy baja (24.5\%), baja (59.2\%) y media (16.3\%). Todas las aves de la zona rural fueron positivas $(n=28)$, mientras que solo el $87.5 \%(21 / 24)$ de las aves de la zona urbana presentaron hemoparásitos, pero sin diferencia significativa entre lugares de procedencia de las aves.

Palabras clave: hemoparásitos; frotis sanguíneo; malaria; palomas

\footnotetext{
${ }^{1}$ Programa de Ecología de Enfermedades y Medicina de la Conservación, Centro de Ornitología y Biodiversidad (CORBIDI), Lima, Perú.

${ }^{2}$ Escuela Académico de Medicina Veterinaria, Facultad de Ciencias Agropecuarias, Universidad Alas Peruanas, Lima, Perú

${ }^{3}$ E-mail: nancy.carlos.erazo@gmail.com
}

Recibido: 12 de setiembre de 2016

Aceptado para publicación: 24 de marzo de 2017 
The aim of the study was to determine the presence of blood parasites in feral populations of the rock dove (Columba livia) in the department of Lima, Peru. The study was carried out in Pampas San Alejo, located in Barranca (rural area) and in a zoo located in San Juan de Miraflores district (urban area). A total of 52 adult birds were captured using mist nets, resulting 28 and 24 pigeons in the rural and urban area respectively. Blood samples from the brachial vein were collected, and blood smears were fixed with methanol and stained with Giemsa. Results showed $94.2 \%(49 / 52)$ positive to the presence of hemoparasites: Haemoproteus sp (94.2\%; 47/52), Plasmodium sp (13.5\%; 7/52) and Leucocytozoon $\mathrm{sp}(1.9 \% ; 1 / 52)$. The Haemoproteus sp infestation intensity was very low (24.5\%), low (59.2\%) and medium (16.3\%). All rural birds were positive, while only $87.5 \%$ $(21 / 24)$ of birds in the urban area had hemoparasites, although this difference was not significant.

Key words: hemoparasites; blood smears; malaria; doves

\section{INTRODUCCIÓN}

El aumento de la densidad poblacional de las palomas de castilla (Columba livia) en áreas urbanas constituye una amenaza para la salud pública. Estas aves son reservorios potenciales de microorganismos patógenos para diferentes especies, la materia fecal representa un riesgo para la salud de los seres humanos, constituyendo una fuente de infección para virus, bacterias y hongos (Tarsitano et al., 2010). Son consideradas como una de las principales fuentes de transmisión de parásitos y enterobacterias para el hombre y animales simpátricos (Pérez et al., 2015).

Un grupo importante de parásitos en estas aves son los hemoparásitos, como los relacionados a la malaria aviar, enfermedad de amplia distribución mundial y transmitida por mosquitos. Esta enfermedad está relacionada con hemosporidios de múltiples linajes de los Plasmodium sp (especialmente $P$. relictum) y Haemoproteus $\mathrm{sp}$. La patogenicidad de la enfermedad ha sido estudiada experimentalmente en varias espe- cies de aves (Atkinson et al., 2000), haciéndose más evidente en aves con infecciones agudas y aves cautivas en zoológicos. Los impactos más significativos de estos parásitos pueden ser patologías subclínicas e indirectas, pudiendo afectar su desarrollo y capacidad reproductiva (Lapointe et al., 2012; Asghar et al., 2015).

Se han realizado estudios sobre los hemoparásitos en C. livia en Latinoamérica, siendo Haemoproteus sp el parásito más frecuente con prevalencias de $29.4 \%$ (Scaglione et al., 2015) hasta 60.5\% (Acosta et al., 2007) y 73\% (Pérez et al., 2015), seguido de Plasmodium sp con $29.4 \%$ y Leucocytozoon sp con $15.7 \%$ (Scaglione et al., 2015). A pesar de las implicancias para la salud que representa C. livia para el Perú, no se encuentran estudios locales sobre hemoparásitos para esta especie.

Debido a la importancia de los hemoparásitos en poblaciones silvestres y con el fin de conocer el papel de la paloma de castilla como reservorio potencial, el objetivo del estudio fue determinar la presencia de hemoparásitos en esta ave en el departamento de Lima. 


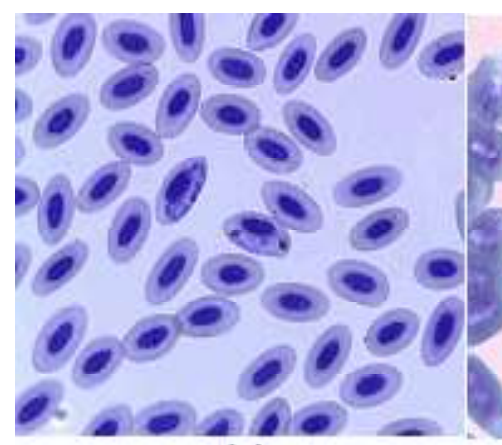

(a) (b)

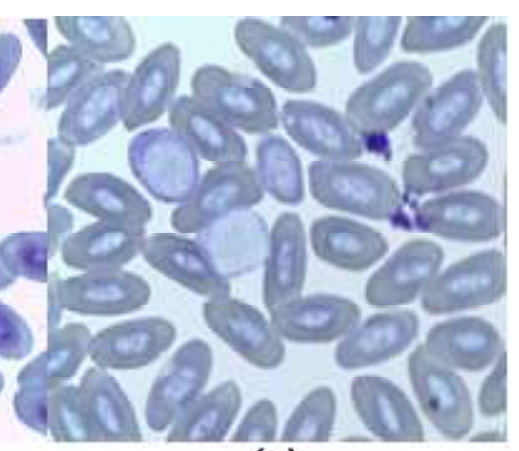

(c)

Figura 1. Hemoparásitos en poblaciones ferales de la paloma de castilla (Columba livia) en el departamento de Lima: a) Haemoproteus sp; b) Plasmodium sp; c) Leucocytozoon sp

\section{Materiales y Métodos}

El estudio se llevó a cabo en una zona urbana y otra rural del departamento de Lima, Perú. La zona urbana correspondió a las áreas verdes del zoológico «Parque Ecológico Campo Santo Santa Rosa», ubicado en el distrito de San Juan de Miraflores, ciudad y provincia de Lima. La zona rural se ubicó en las áreas de cultivo del centro poblado Pampa San Alejo, ubicado en la ciudad y provincia de Barranca. El estudio se llevó a cabo bajo la Resolución $N^{\circ}$ 3310-2014-FCA-EPMVUAP, emitida por la Facultad de Ciencias Agropecuarias de la Universidad Alas Peruanas.

Se capturaron 52 aves adultas, utilizando cuatro redes de neblina de 8 y $12 \mathrm{~m}$ de largo, ubicadas en zonas donde las aves solían bajar a buscar comida. Las redes fueron abiertas por $4 \mathrm{~h}$ al día (10:00 - 14:00) por $7 \mathrm{~d}$ en cada zona. Las aves fueron pesadas con una balanza electrónica (BSH 600 PCE®), y se tomaron las medidas biométricas (tarso y largo de ala y cola) con la ayuda de un pie de rey y una cintra métrica.

Se tomaron muestras de sangre $(0.1 \mathrm{ml})$ de la vena braquial de cada ave. Para esto, las aves fueron colocadas en posición laterolateral, colocando el ala en un ángulo de $90^{\circ}$ con el eje corporal. Se realizó el frotis sanguíneo de cada muestra, dejándolo secar a temperatura ambiente, para luego ser fijado con metanol.

El análisis de laboratorio se llevó a cabo en la Universidad Alas Peruanas, Lima. Las láminas fueron teñidas con Giemsa y observadas en el microscopio con objetivo 100X. Para determinar la intensidad de infestación para Haemoproteus sp se contabilizaron los elementos parasitarios examinando 100000 glóbulos rojos. Se consideró como «muy baja» infección la observación de menos de 11 elementos, «baja» con 11 a 100 elementos, «medio» con 101 a 1000 elementos, «alta» con 1001 a 10000 elementos y «muy alta» con más de 10000 elementos (Garvin et al., 1993, Benedikt et al., 2009). Para la identificación de las especies de parásitos hematófagos se consideró lo descrito por Valkiu (1997), Tietz et al. (2007) y Atkinson y Hunter (2008).

Los datos se analizaron de manera porcentual. Para evaluar las posibles diferencias según el lugar de procedencia se utilizó la prueba Exacta de Fisher $(\mathrm{p}<0.05)$, utilizando el programa estadístico con RStudio v. 0.99.903@ (https://www.rstudio.com/). 


\section{Resultados}

El 94.2\% (49/52) de las aves fueron positivas a hemoparásitos, identificándose tres especies: Haemoproteus sp, Plasmodium sp y Leucocytozoon sp (Figura 1). En el Cuadro 1 se muestran la frecuencia de los parásitos encontrados según la procedencia, siendo de 100 y $87.5 \%$ para las zonas urbana y rural, respectivamente, sin diferencia estadística entre zonas $(\mathrm{p}=0.09)$.
La infestación más frecuente fue de grado medio $(59.2 \%)$, con diferencia significativa entre la zona rural y urbana $(\mathrm{p}=0.013)$ (Cuadro 2). Además, se observó monoparasitismo (Haemoproteus $\mathrm{sp}$ ) en el $80.8 \%(42 / 52)$ de las aves, seguido de biparasitismo (Haemoproteus sp y Plasmodium sp) con $11.5 \%(6 / 52)$ y triparasitismo (Haemoproteus sp, Plasmodium sp y Leucocytozoon sp) con $1.9 \%$ (1/52) (Cuadro 3).

Cuadro 1. Hemoparásitos en poblaciones ferales de la paloma de castilla (Columba livia) según lugar de procedencia (Lima, Perú)

\begin{tabular}{|c|c|c|c|c|c|c|c|c|c|}
\hline \multirow{2}{*}{ Zona } & \multirow{2}{*}{ N. ${ }^{\circ}$} & \multicolumn{2}{|c|}{ Haemoproteus sp } & \multicolumn{2}{|c|}{ Plasmodium sp } & \multicolumn{2}{|c|}{ Leucocytozoon sp } & \multicolumn{2}{|c|}{ Total } \\
\hline & & $\mathrm{n}$ & $\%$ & $\mathrm{n}$ & $\%$ & $\mathrm{n}$ & $\%$ & $\mathrm{n}$ & $\%$ \\
\hline Urbano & 24 & 21 & 87.5 & - & - & - & - & 21 & $87.5^{\mathrm{a}}$ \\
\hline Rural & 28 & 28 & 100.0 & 7 & 25.0 & 1 & 3.57 & 28 & $100.0^{\mathrm{a}}$ \\
\hline Total & 52 & 49 & 94.2 & 7 & 13.5 & 1 & 1.92 & 49 & 94.2 \\
\hline
\end{tabular}

${ }^{a}$ Superíndices similares dentro de columnas indican ausencia de diferencia significativa

Cuadro 2. Intensidad de infestación ${ }^{1}(\%)$ de Haemoproteus sp en poblaciones ferales de la paloma de castilla (Columba livia) en el departamento de Lima, Perú

\begin{tabular}{ccccc}
\hline Zona & $\begin{array}{c}\text { Positivos } \\
(\mathrm{n})\end{array}$ & Muy baja & Baja & Media \\
\hline Urbana & 21 & $42.9^{\mathrm{a}}$ & 47.6 & 9.5 \\
Rural & 28 & $10.7^{\mathrm{b}}$ & 67.9 & 21.4 \\
\hline Total & 49 & 24.5 & 59.2 & 16.3 \\
\hline
\end{tabular}

${ }^{1}$ Muy baja:<11; Baja: 11 a 100; Media: 101 a 1000 elementos parasitarios en 100000 glóbulos rojos

$a, b$ Superíndices diferentes dentro de columnas indican diferencia significativa $(p<0.05)$ 
Cuadro 3. Infecciones de hemoparásitos únicas y mixtas en poblaciones ferales de la paloma de castilla (Columba livia) en el departamento de Lima, Perú

\begin{tabular}{lcc}
\hline \multirow{2}{*}{ Parasitismo } & \multicolumn{2}{c}{ Positivos } \\
\cline { 2 - 3 } & $\mathrm{n}$ & $\%$ \\
\hline Monoparasitismo (Haemoproteus sp) & 42 & 80.8 \\
Biparasitismo (Haemoproteus sp y Plasmodium sp) & 6 & 11.5 \\
Triparasitismo (Haemoproteus, Plasmodium, Leucocytozoon) & 1 & 1.9 \\
\hline Total & 49 & 94.2 \\
\hline
\end{tabular}

\section{Discusión}

El estudio de los hemoparásitos brinda información importante sobre los aspectos ecológicos y de comportamiento del hospedero, permitiendo evaluar el efecto de estos parásitos y su aplicación en la conservación de especies de vida silvestre (Matta y Rodríguez, 2001). El hallazgo de tres especies de hemoparásitos son relevantes, no solo para la paloma de castilla (C. livia), sino, también, para las aves simpátricas que podrían ser afectadas.

La frecuencia de $94.2 \%$ de palomas infectadas es la más alta reportada para la especie en Sudamérica, por encima del 67.2\% hallado en Brasil (Tietz et al., 2007), del 73\% en Colombia (Pérez et al., 2015), del 71.4\% en Cuba (Acosta et al., 2007) y del 55.6\% en Irán (Gupta et al., 2011). La alta prevalencia de hemoparásitos se puede deber a la presencia de los vectores de transmisión como son Pseudolynchia canariensis para Haemoproteus sp (Soulsby, 1987; Acosta et al., 2007), los mosquitos de la familia Culicidae para Plasmodium sp (Saif, 2008) y mosquitos de la familia Simuliidae para Leucocytozoon sp (Lotta,2010). Además, la alta prevalencia de hemoparásitos depende de la susceptibilidad del hospedero, edad y hábitat (Scaglione et al., 2015). Adicional- mente, la presencia de hemoparásitos se ha asociado a los periodos de cría y al estrés de las aves en etapa reproductiva (Matta y Rodríguez, 2001).

Los hemoparásitos hallados en el estudio de los géneros Haemoproteus, Plasmodium y Leucocytozoon los grupos más frecuentes en el mundo (Matta y Rodríguez, 2001). Haemoproteus sp fue el de mayor frecuencia (94.2\%), similar al estudio de Tietz et al. (2007) en Brasil (67.2\%), y el único hemoparásito encontrado en Colombia en esta especie (Pérez et al., 2015). En otras especies aviares como es el caso de aves Strigiformes y Falconiformes se reportan porcentajes similares (67\%) (Forrester $e t$ $a l ., 1994)$. Además, el vector $P$. canariensis, al ser un ectoparásito cosmopolita, puede fácilmente transmitir el hemoparásito, pudiendo infectar a otras especies aviares (Adriano y Cordeiro, 2001; Gicik y Arslan, 2001). En circunstancias normales, especies del género Haemoproteus se consideran no patógenas, pero cuando la parasitemia es intensa puede causar problemas clínicos si el ave está estresada o inmunosuprimida (Ritchie y Harrison, 1997).

La presencia de Plasmodium sp en este estudio (13.5\%) fue menor a la reportada en otros países como India con 55.6\% (Jahan et 
al., 2011), Irak con 36.6\% (Al-Barwari y Saeed, 2012) e Italia con $29.4 \%$ (Scaglione et al., 2015). En el Perú, un estudio halló Plasmodium relictum en el $32.4 \%$ de aves Passeriformes de los departamentos de Huánuco y Lima (Marzal et al., 2014). El género Plasmodium está distribuido globalmente y puede infectar a cientos de especies aviares. La mayor diversidad de especies de Plasmodium sp ha sido documentada en Galliformes, Columbiformes y Passeriformes (Ritchie y Harrison, 1997). La variabilidad puede deberse a los factores ecológicos propios de cada lugar, como el hábitat, clima, presencia del vector, susceptibilidad del ave y el contacto con aves migratorias infectadas (Scaglione et al., 2015). Los plasmodios no son patógenos para sus hospedadores naturales, pero sí podrían serlo para los que no son habituales; por ejemplo, los animales de parques zoológicos (Borchert, 1981), o en el eventual encuentro de nuevos hospederos producto de variaciones de las condiciones ecológicas o ambientales de sus vectores (Loiseau et al., 2013).

Leucocytozoon sp fue el hemoparásito de menor presentación en este estudio $(1.9 \%)$, similar a valores reportados en otras latitudes (Borji et al., 2011; Nath et al., 2014). Se le encuentra en baja prevalencia en el Neotrópico, hallándose mayormente en el Neártico y Paleártico. Además, se relaciona con la escasa presencia de vectores ornitofílicos apropiados (familia Simuliidae) (Mata y Rodríguez, 20013). La infección se debería a la presencia de aves migratorias infectadas (Rodríguez et al., 2009). Este hemoparásito causa destrucción directa de los hematíes parasitados, tanto en la médula ósea como en la sangre circulante (Cordero del Campillo et al., 1999).

No se encontró diferencias en la frecuencia de parasitismo entre las zonas urbana y rural en estudio, indicando que las palomas tienen las mismas posibilidades de ser infectadas. Haemoproteus sp fue prevalente en ambas zonas, evidenciando solo al vector $P$. canariensis en cuatro aves de la zona ur- bana. Otro posible vector serían los mosquitos Culicoide (Sousbly, 1987; Cardoso, 2010), especialmente en zonas rurales con cultivos y regadíos (García et al., 2011). Plasmodium sp solo se evidenció en la zona rural, posiblemente por la presencia de los vectores Culex spp y Aedes spp de la familia Culicidae (Campbell, 1995).

\section{Conclusiones}

Se evidencia una alta prevalencia de hemoparásitos en la paloma castilla (Columba livia) del departamento de Lima (94.23\%), identificando Haemoproteus sp (94.2\%), Plasmodium sp (13.5\%) y Leucocytozoon $\mathrm{sp}(1.9 \%)$, sin diferencias entre las dos zonas del estudio.

\section{Agradecimiento}

A las MV Eva Chomba y Elizabeth Solano por su colaboración en la captura de las aves.

\section{Literatura Citada}

1. Acosta I, Soto C, Cruz E. 2007. Prevalencia de Haemoproteus spp en palomas. Rev Cubana Cienc Avícola 31: 107112.

2. Adriano E, Cordeiro N. 2001. Prevalence and intensity of Haemoproteus columbae in three species of wild doves from Brazil. Mem Inst Oswaldo Cruz 96: 175-178. doi: http:// dx.doi .org/10.1590/S 0074 02762001000200007

3. Al-Barwari S, Saeed I. 2012. The parasitic communities of the rock pigeon Columba livia from Iraq: component and importance. Turkiye Parazitol Derg 36: 232-239. doi: 10.5152/tpd.2012.56

4. Asghar M, Hasselquist D, Hansson B, Zehtindjiev P, Westerdahl H, Bensch S. 2015. Hidden costs of infection: chronic malaria accelerates telomere 
degradation and senescence in wild birds. Science 23: 436-438. doi: 10.1126/ science. 1261121

5. Atkinson CT, Hunter B. 2008. Parasitic diseases of wild birds. New Jersey, USA: Wiley-Blackwell. 592 p.

6. Atkinson CT, Dusek RJ, Woods KL, Iko WM. 2000. Pathogenicity of avian malaria in experimentally-infected Hawaii Amakihi. J Wild Dis 36: 197-204. doi: 10.7589/0090-3558-36.2.197

7. Benedikt V, Barus V, Capek M, Havlicek M, Literak I. 2009. Blood parasites (Haemoproteus and microfilariae) in birds from the Caribbean slope of Costa Rica. Acta Parasito 54: 197204. doi: 10.2478/s11686-009-0043-1

8. Borchert A. 1981. Parasitología veterinaria. $3^{\mathrm{a}}$. ed. Zaragoza: Acribia. 745 p.

9. Borji H, Moghaddas E, Razmi G, Heidarpour M, Mohri M, Azad M. 2011. Prevalence of pigeon haemosporidians and effect of infection on biochemical factors in Iran. J Parasit Dis 35: 199-201. doi: 10.1007/s12639-0110056-1

10. Campbell T. 1995. Avian hematology and cytology. $2^{\text {nd }}$ ed. Iowa State: Iowa State University Press. 108 p.

11. Cardoso G 2010. Haemoproteus sp e Plasmodium sp em pombos domésticos (Columba livia domestica). Revisão bibliográfica e relato de caso. São Paulo: Universidade Castelo Branco. $44 \mathrm{p}$.

12. Cordero del Campillo M, Rojo F, Martínez A, Sánchez M, Hernández S, Navarrete I, Diez P. et al. 1999. Parasitología veterinaria. Barcelona: McGraw-Hill Interamericana. $450 \mathrm{p}$.

13. Forrester D, Telford S; Foster G. 1994. Blood parasites of raptors in Florida. $\mathrm{J}$ Raptor Res 28: 226-231.

14. García M, García T, Mellano M, Villota J. 2011. Patología infecciosa importada I: malaria. En: Protocolos diagnósticos y terapéuticos de la AEP: Infectología pediátrica. Barcelona: ERGON. p 221-229.
15. Garvin M, Remsen J, Bishop $M$, Bennett G. 1993. Hematozoa from passeriform birds in Louisiana. J Parasitol 79:318-321. doi: 10.2307/3283564

16. Gicik Y, Arslan Ö. 2001. Blood parasites of wild pigeons in Ankara district. Turk J Vet Anim Sci 25: 169-172.

17. Gupta D, Jahan N, Gupta N. 2011. Distribution pattern of apicomplexan parasites (Sporozoa: Haemosporida) in Columba livia, Gmelin. J Parasit Dis 35: 18-22. doi: 10.1007/s12639-011-0026-7

18. Jahan N, Chandra R, Shoeb M. 2011. Parasitimic load of haematozoan parasites in rock pigeons (Columba livia). Rec Res Sci Tech 3(6): 9-11.

19. LaPointe D, Atkinson C, Samuel M. 2012. Ecology and conservation biology of avian malaria. Ann NY Acad Sci 1249: 211-226. doi: 10.1111/j.1749-6632.2011.06431.x

20. Loiseau C, Harrigan RJ, Bichet $C$, Julliard R, Garnier S, Lendvai AZ, Chastel O, Sorci G 2013. Predictions of avian Plasmodium expansion under climate change. Sci Rep 24: 1126. doi: 10.1038/srep01126

21. Lotta I. 2010. Presencia de simúlidos ornitofílicos en el Parque Nacional Natural (PNN) Chingaza: implicaciones en la transmisión del hemoparásito Leucocytozoon sp. Tesis de Magíster. Bogotá: Univ Nacional de Colombia. 109 p.

22. Matta N, Rodríguez O. 2001. Hemoparásitos aviares. Acta Biol Colomb 6(1): 27-34.

23. Marzal A, Garcia-Longoria L, Cardenas J, Sehgal R. 2014. Invasive avian malaria as an emerging parasitic disease in native birds of Peru. Biol Invasions 17: 39-45. doi: 10.1007/s10530014-0718-x

24. Nath T, Bhuiyan M, Alam M. 2014. A study on the presence of leucocytozoonosis in pigeon and chicken of hilly districts of Bangladesh. Biol Sci Pharmacol Res 2(2): 13-18.

25. Pérez J, Monsalve D, Márquez C. 2015. Presencia de parásitos y enterobacterias en palomas ferales 
(Columba livia) en áreas urbanas en Envigado, Colombia. Rev Fac Nac Salud Pública 33: 370-376. doi: 10.17533/ udea.rfnsp.v33n3a06

26. Ritchie B, Harrison G 1997. Avian medicine: principles and applications. Florida: Wingers Publishing. 809 p.

27. Rodriguez O, Moya H, Matta, N. 2009. Parásitos sanguíneos de aves en el Parque Nacional Natural Chingaza: Andes de Colombia. Hornero 24(1): 1-6.

28. Saif Y. 2008. Diseases of poultry. $12^{\text {th }}$ ed. Iowa, USA: Blackwell Publishing. $1409 \mathrm{p}$.

29. Scaglione F, Pregel P, Tiziana F, Pérez A, Ferroglio E, Bollo E. 2015. Prevalence of new and known species of haemoparasites in feral pigeons in northwest Italy. Malaria J 14: 99. doi: 10.1186/s12936-015-0617-3

30. Soulsby E. 1987. Parasitología y enfermedades parasitarias en los animales domésticos. $7^{\mathrm{a}}$ ed. México DF: Ed interamericana. $823 \mathrm{p}$.
31. Talavera M. 2011. Factores que condicionan la sobrepoblación de palomas. En: IV Congreso Latinoamericano de Estudiantes de Ciencias Veterinarias. Huánuco: Univ Nacional Hermilio Valdizán de Huánuco.

32. Tarsitano E, Greco G, Decaro N, Nicassio F, Lucente M, Buonavoglia C. et al. 2010. Environmental monitoring and analysis of faecal contamination in an urban setting in the city of Bari (Apulia Region, Italy): health and hygiene implications. Int J Environ Res Public Health 7: 3972-3986. doi: 10.3390/ ijerph7113972

33. Tietz S, Marinho de Cuadros R, Jardim da Silva C, Baldo M. 2007. Parasites of pigeons (Columba livia) in urban areas of lages, Southern Brazil. Parasitol Latinoam 62: 83-187. doi: http:/ /dx.doi.org/10.4067/S0717 77122007000200014

34. Val'kiunas G 1997. Bird haemosporida. Acta Zoologica Lituanica 3-5: 1-607. 